\title{
SEQUENCE ANALYSIS OF SCHMALLENBERG VIRUS GENOMES DETECTED IN HUNGARY
}

\author{
ENIKŐ FEHÉR ${ }^{1}$, SZILVIA MARTON ${ }^{1}$, ÁDÁM GYÖRGY TÓTH ${ }^{2}$, KRISZTINA URSU ${ }^{3}$, \\ KERSTIN Wernike ${ }^{4}$, MARTIN BEER ${ }^{4}$, ÁdÁm DÁN ${ }^{3}$ and KRISZTIÁN BÁNYAI ${ }^{*}$ \\ ${ }^{1}$ Institute for Veterinary Medical Research, Centre for Agricultural Research, Hungarian \\ Academy of Sciences, Budapest, Hungary \\ ${ }^{2}$ Ceva-Phylaxia Co. Ltd., Budapest, Hungary \\ ${ }^{3}$ National Food Chain Safety Office, Veterinary Diagnostic Directorate, Budapest, \\ Hungary \\ ${ }^{4}$ Institute of Diagnostic Virology, Friedrich-Loeffler-Institut, Greifswald, Germany
}

(Received: 6 June 2017; accepted: 24 August 2017)

Since its emergence near the German-Dutch border in 2011, Schmallenberg virus (SBV) has been identified in many European countries. In this study, we determined the complete coding sequence of seven Hungarian SBV genomes to expand our knowledge about the genetic diversity of circulating field strains. The samples originated from the first case, an aborted cattle fetus without malformation collected in 2012, and from the blood samples of six adult cattle in 2014. The Hungarian SBV sequences shared $\geq 99.3 \%$ nucleotide (nt) and $\geq 97.8 \%$ amino acid (aa) identity with each other, and $\geq 98.9$ nt and $\geq 96.7 \%$ aa identity with reference strains. Although phylogenetic analyses showed low resolution in general, the $\mathrm{M}$ sequences of cattle and sheep origin SBV strains seemed to cluster on different branches. Both common and unique mutation sites were observed in different groups of sequences that might help understanding the evolution of emerging SBV strains.

Keywords: Schmallenberg virus, genome, sequence, mutations, cattle, sheep

\section{Introduction}

Members of the Orthobunyavirus genus (family Peribunyaviridae), which includes 48 virus species and a number of unclassified isolates, are widely distributed arthropod-transmitted viruses and may cause severe economic losses in animal husbandry and serious infections in human [1]. Schmallenberg virus (SBV), a recently emerged pathogen, belongs to the Simbu serogroup along with Akabane virus, Aino virus, Shamonda virus, and Sathuperi virus [2, 3]. The SBV

\footnotetext{
*Corresponding author; E-mail: bkrota@hotmail.com
} 
genome is composed of three single-stranded RNA segments with negativesense orientation. The large (L) segment encodes the RNA-dependent RNA polymerase. The medium (M) segment encodes a precursor protein, which is posttranslationally processed to yield the envelope glycoproteins $\mathrm{Gn}$ and $\mathrm{Gc}$ and a non-structural protein NSm [4]. An N-terminal hypervariable region was identified in the Gc region, which is the main target of neutralizing antibodies, and may facilitate early events in the SBV infection process and host cell protein shutoff [5-7]. The small (S) segment encodes the overlapping open reading frames (ORFs) of nucleocapsid $(\mathrm{N})$ and small non-structural (NSs) proteins, which may interact with cellular interferon production [8-10].

SBV was the first member of the Simbu serogroup detected in Europe. The epidemics swept through the European continent in 2011-2012 and reemerged in $2014[11,12]$. The presence of viral genome has been confirmed in various ruminants (cattle, sheep, goat, elk, and wildebeest) and serology tests indicated that a number of additional species (bison, deer, mouflon, and chamois) are also susceptible to SBV infection [13-17]. In adult hosts, SBV infection typically results in subclinical infection or moderate clinical signs, such as fever, decreased milk production, or diarrhoea. However, in pregnant animals, SBV has been reported to cause congenital malformations, miscarriage, and stillbirth [18-20]. Transmission of SBV occurs by Culicoides biting midges similar to related viruses of the Peribunyaviridae [21-23].

In this study, we sequenced and analyzed seven near complete SBV genomes originating from infected cattle, including the first described case (an aborted but not malformed calf) in Hungary, 2012, and selected blood samples collected in 2014 when additional cases were identified during epidemiological surveillance.

\section{Materials and Methods}

Ethylenediaminetetraacetic acid anticoagulated whole blood and tissue samples of cattle and small ruminants have regularly been submitted since May 2012 to the Veterinary Diagnostic Directorate of the National Food Chain Safety Office, Budapest, Hungary, for diagnostic purposes and to confirm SBV-free status required for trading.

RNA extraction was carried out using the MagAttract Virus Mini M48 Kit (Qiagen, Hilden, Germany) on a KingFisher 96 Flex instrument (Thermo Scientific, Waltham, MA, USA) according to the manufacturer's instructions.

The samples were analyzed by a previously described S-segment-based real-time reverse transcription-polymerase chain reaction (RT-PCR) [24]. Confirmation of positive test results was done by sequencing of PCR products 
amplified by a conventional RT-PCR method (primer sequences and protocol available upon request).

Fragments of the SBV S and M segments were amplified using the primer sets designed by Fischer et al. [5], whereas PCR products covering the complete L segment were generated by new primer sets (Table I). RT-PCR assays were performed using the QIAGEN OneStep RT-PCR Kit (Qiagen, Hilden, Germany). The RT-PCR thermal profile composed of the RT step at $50{ }^{\circ} \mathrm{C}$ for $30 \mathrm{~min}, \mathrm{PCR}$ initial activation step at $95^{\circ} \mathrm{C}$ for $15 \mathrm{~min}$, and 45 cycles of the amplification steps including denaturation at $94{ }^{\circ} \mathrm{C}$ for $30 \mathrm{~s}$, primer annealing $\left(55^{\circ} \mathrm{C}\right.$ for the $\mathrm{S}$ and $\mathrm{M}$ and $50{ }^{\circ} \mathrm{C}$ for the $\mathrm{L}$ segment) for $30 \mathrm{~s}$ and the elongation at $72{ }^{\circ} \mathrm{C}$ for $2 \mathrm{~min}$, and the final elongation step at $72{ }^{\circ} \mathrm{C}$ for $10 \mathrm{~min}$.

The gel-purified PCR products (Gel/PCR DNA Fragments Extraction kit, Geneaid Biotech Ltd., New Taipei City, Taiwan) were mixed and used for library preparation with the NEBNext ${ }^{\circledR}$ Fast DNA Fragmentation \& Library Prep Set for Ion Torrent (New England BioLabs Inc., Ipswich, MA, USA) and the Ion Torrent Xpress Barcode Adapters (ThermoFisher Scientific, Waltham, MA, USA). The emulsion PCR and templated bead enrichment were carried out using the OneTouch v2 instrument and Ion OneTouch ${ }^{\mathrm{TM}}$ ES (ThermoFisher Scientific, Waltham, MA, USA). Sequencing was performed with a 316 chip using the Ion Torrent Personal Genome Machine ${ }^{\circledR}$ (ThermoFisher Scientific, Waltham, MA, USA). Sequences were assembled and aligned with CLC Genomics Workbench version 7 software (http://www.clcbio.com). The newly determined SBV genome sequences were deposited in GenBank (KX384856-KX384876).

The assembled genomes were aligned with the AliView software [25]. MEGA6 software was applied for the preparation of maximum likelihood phylogenetic trees generated with the best-fit models and for sequence comparisons [26]. For sequence comparison, a set of reference strains were obtained from GenBank (Table II).

Table I. Primer sequences used for the amplification of $\mathrm{L}$ segments of SBV strains

\begin{tabular}{lc}
\hline Primer name & \multicolumn{1}{c}{ Primer sequence $\left(5^{\prime}-3^{\prime}\right)$} \\
\hline SBVL-F1 & AGTAGTGTACCCCTAATTACAATCAC \\
SBVL-R2345 & TGGTCCCTTTCTATATCTAAGATTGTC \\
SBVL-F2183 & ATAACAGAGACCTATCATCAATCTGG \\
SBVL-R4620 & CTTCTTGCCAATAATACATGTAGCATC \\
SBVL-F4488 & AGCTCAGCTCTTCATAGAACAAG \\
SBVL-R6882 & AGTAGTGTGCCCCTAATTACATG \\
\hline
\end{tabular}


Table II. Accession number and name of SBV reference strains used in this study

\begin{tabular}{|c|c|c|c|c|c|}
\hline Strain & Host & S segment & M segment & L segment & Reference \\
\hline BH80/11-4 & Cattle & HE649914 & HE649913 & HE649912 & [1] \\
\hline BH02/12-1 & Lamb & KC108842 & KC108843 & - & [5] \\
\hline $\mathrm{BH} 03 / 12-3$ & Lamb & KC108844 & KC108845 & - & \\
\hline BH28/12-5 & Lamb & $\mathrm{KC} 108846$ & KC108847 & - & \\
\hline ВН37/12-2 & Sheep & $\mathrm{KC} 108848$ & KC108849 & - & \\
\hline BH59/12-8 & Sheep & KC108850 & KC108851 & - & \\
\hline BH77/12-1 & Lamb & $\mathrm{KC} 108852$ & KC108853 & - & \\
\hline BH127/12-16 & Lamb & КC108854 & KC108855 & - & \\
\hline BH148/12-9 & Lamb & KC108856 & KC108857 & - & \\
\hline BH174/12-2 & Lamb & KC108858 & KC108859 & - & \\
\hline BH197/12-3 & Sheep & КC108860 & KC108861 & - & \\
\hline BH198/12-5 & Sheep & KC108862 & KC108863 & - & \\
\hline BH199/12-5 & Lamb & KC108864 & KC108865 & - & \\
\hline BH200/12-2 & Sheep fetus & КC108866 & KC108867 & - & \\
\hline BH231/12-1 & Sheep fetus & KC108868 & KC108869 & - & \\
\hline BH233/12-1 & Goat & KC108870 & KC108871 & - & \\
\hline BH237/12-4 & Lamb & KC108872 & KC108873 & - & \\
\hline BH248/12-1 & Cattle & KC108874 & KC108875 & - & \\
\hline BH250/12-2 & Cattle & $\mathrm{KC} 108876$ & KC108877 & - & \\
\hline BH336/12-1 & Sheep & KC108878 & KC108879 & - & \\
\hline BH336/12-3 & Sheep & KC108880 & KC108881 & - & \\
\hline BH635/12-2 & Cattle & КC108884 & KC108885 & - & \\
\hline $\mathrm{Na} 1$ & Sheep fetus & KC139376 & KC139368 & KC139362 & [27] \\
\hline $\mathrm{Na} 2$ & Sheep fetus & KC139379 & KC139372 & KC139365 & \\
\hline HL1 & Lamb & KC355456 & KC355455 & КC355454 & {$[28]$} \\
\hline F6 & Cattle & КC355459 & КC355458 & КC355457 & \\
\hline BH619/12 & Sheep & KP731865 & KP731871 & KP731881 & [12] \\
\hline BH652/12 & Cattle & KP731866 & KP731872 & KP731880 & \\
\hline D495/12-1 & Cattle & KP731867 & KP731873 & KP731879 & \\
\hline BH119/14-1/2 & Cattle & KP731868 & KP731874 & KP731877 & \\
\hline BH119/14-3/4 & Cattle & KP731869 & KP731875 & KP731878 & \\
\hline BH132/14 & Cattle & KP731870 & KP731876 & KP731882 & \\
\hline 79.4 & Cattle & - & KM047423 & KM047416 & [29] \\
\hline 91.1 & Cattle & - & KM047424 & KM047417 & \\
\hline 96.1 & Cattle & - & KM047425 & KM047418 & \\
\hline 100.3 & Cattle & - & KM047426 & KM047419 & \\
\hline 102.2 & Cattle & - & KM047427 & KM047420 & \\
\hline 175.2 & Cattle & - & KM047428 & KM047421 & \\
\hline 200.2 & Cattle & - & KM047429 & KM047422 & \\
\hline SBV/2013/TR/Krkl.1 & Cattle & KP279304 & - & - & \\
\hline
\end{tabular}

Note: SBV: Schmallenberg virus.

\section{Results}

Since the introduction of an SBV-specific real-time RT-PCR screening method in routine diagnostics in May 2012, more than 100,000 samples have been 
analyzed at the Veterinary Diagnostic Directorate to date. The great majority (98.6\%) of the examined samples were whole blood samples sent by trading companies to obtain an SBV-free certificate for trading purposes. The remaining samples were different tissue samples (mainly brain) sent from cattle and small ruminant abortion cases where SBV infection was suspected. Samples originating from cattle (98.4\% whole blood samples and $78.4 \%$ tissue samples) were overrepresented compared with that from small ruminants.

The first SBV infection was detected in the brain tissue specimen of an aborted calf received in October 2012. In October and November 2014, whole blood samples from 18 cattle originating from five different cattle farms were tested positive of SBV. Genome sequencing was performed on seven Hungarian SBV strains. The strains selected for sequencing included the first Hungarian record of SBV infection in 2012 and representative strains from the 2014 outbreak. In particular, SBV-Hun4 was the strain detected from an aborted calf in 2012, whereas SBV-Hun1-3 and SBV-Hun5-7 were identified in blood samples of independent acute cases that originated from four different farms.

Low degree of variability was observed in the 798 nucleotide (nt) long S-segment sequences of the Hungarian SBV strains. The alignment revealed $32 \mathrm{nt}$ substitutions (one in the stop codon of a lamb sequence) and five amino acid (aa) mutation sites in the 702-nt long N protein coding ORF of the 47 investigated SBV $\mathrm{S}$ segments (18 cattle, 21 sheep, and 1 goat sequence, including the seven Hungarian strains) (Tables II and III). Four unique synonymous nt mutation sites were observed in the Hungarian sequences. One of these, the substitution A276G in the ORF, was present in more than one sequence (SBV-Hun1, SBV-Hun3, SBV-Hun5, and SBV-Hun6), whereas the other three were single nt mutations of variable sequences. An additional nt change (G755A) was detected in the $3^{\prime}$ non-coding region (NCR) of the strain SBV-Hun1. The nt and aa identities ranged between $99.4 \%-99.6 \%$ and $99.1 \%-100 \%$, respectively, among the

Table III. Nucleotide (nt) and amino acid (aa) mutation sites detected in the coding sequence of Hungarian and reference SBV genomes

\begin{tabular}{lcccc}
\hline & N ORF (nt/aa) & NSs ORF (nt/aa) & M ORF (nt/aa) & RdRp ORF (nt/aa) \\
\hline $\mathrm{C}$ & $5 / 0$ & $2 / 2$ & $70 / 27$ & $87 / 30$ \\
$\mathrm{~S}$ & $24 / 3$ & $17 / 11$ & $232 / 139$ & $26 / 11$ \\
$\mathrm{G}$ & $2 / 1$ & $2 / 2$ & $13 / 6$ & $0 / 0$ \\
$\mathrm{C}+\mathrm{S}$ & $1 / 1$ & $0 / 0$ & $19 / 18$ & $4 / 0$ \\
$\mathrm{C}+\mathrm{G}$ & $0 / 0$ & $0 / 0$ & $4 / 7$ & $0 / 0$ \\
$\mathrm{C}+\mathrm{S}+\mathrm{G}$ & $0 / 0$ & $0 / 0$ & $0 / 1$ & $0 / 0$ \\
\hline
\end{tabular}

Note: C: cattle; S: sheep; G: goat; ORF: open reading frame; SBV: Schmallenberg virus; RdRp: RNA-dependent RNA polymerase. 
investigated Hungarian and GenBank SBV sequences. Shamonda virus (accession number: HE795107) was the closest relative (97.4\%-97.7\% nt and $100 \%$ aa identity) within the $\mathrm{N}$ protein coding region of the $\mathrm{S}$ segments.

In total, $21 \mathrm{nt}$ and 15 aa mutation sites (three in the premature stop codon of truncated sheep sequences) were identified within the 44 complete and three truncated (BH02/12-1, BH37/12-2, BH77/12-1) NSs sequences of different origin SBV strains (Tables II and III) [5]. Two sites (A74G and A251G) identified in cattle's NSs region were unique for the 276-nt long Hungarian sequences and caused aa changes (N25S and H84R) in one (SBV-Hun2) and four (SBV-Hun1, SBV-Hun3, SBV-Hun5, and SBV-Hun6) sequences, respectively. The Hungarian strains showed $99.3 \%-100 \%$ nt and $97.8 \%-100 \%$ aa identity with each other, and $98.9 \%-100 \%$ nt and $96.7 \%-100 \%$ aa identity with other SBV reference strains. The $n t$ and aa identities were $98.5 \%-98.9 \%$ and $95.6 \%-96.7 \%$ among the NSs sequences of SBVs and the Shamonda virus, respectively.

The sequenced 4,365- (SBV-Hun1) and 4,373-nt (SBV-Hun2-7) long fragments of the M segment of Hungarian SBVs included the 4,212-nt long ORF. A unique A8T nt substitution was identified in the $5^{\prime} \mathrm{NCR}$ of strains SBV-Hun2-7. The partial 3' NCR of the SBV-Hun7 M sequence, similar to two sequences obtained from GenBank (strains 200.2 and 79.4), included nt substitution at position A4292G. In total, $338 \mathrm{nt}$ and 198 aa mutation sites were found in the ORF region of M segment (24 cattle, 21 sheep, and 1 goat) (Tables II and III). About $31 \mathrm{nt}$ (11 non-synonymous) mutation sites were found in the Hungarian sequences out of which 22 were unique and caused aa substitutions at seven positions. At four nt sites (C285T, C609T, C623T, and T1569C), changes occurred in multiple Hungarian SBV sequences. The non-synonymous C623T substitution (resulting T208M in the deduced protein sequence) was identified in SBV-Hun1, SBV-Hun3, SBV-Hun5, and SBV-Hun6 sequences, whereas the synonymous T1569C substitution was identified in the $\mathrm{M}$ sequence of these four Hungarian SBVs and the Swiss strain 96.1. The synonymous substitutions C285T and C609T were detected in the M sequence of strain SBV-Hun4 and SBV-Hun7, and SBV-Hun2 and SBV-Hun7, respectively. The C285T change also appeared in nine other cattle and sheep SBV genomes, whereas the latter was unique for the Hungarian sequences. The nt and aa identity was $99.5 \%-100 \%$ among the Hungarian $M$ sequences, which showed $99.2 \%-100 \%$ nt and $98.4 \%-100 \%$ aa identity with the reference SBV sequences. The M segment of SBVs showed $82.1 \%-82.3 \%$ nt and $89.7 \%-89.9 \%$ aa identity with the corresponding segment of the closest relative, a Sathuperi virus strain (accession number: AB698474).

The complete $\mathrm{L}$ segments were determined for all seven Hungarian SBV strains. The structure of the 6,882-nt long segments was comparable with those SBV strains we used for comparison. In total, $117 \mathrm{nt}$ and 41 aa mutation sites have 
been found in the 6,765-nt long coding region ( 21 cattle and 4 sheep sequences) (Tables II and III), and a single nt change was found in the 3' NCR of a reference strain (BH619/12). About $52 \mathrm{nt}$ (44 unique) and 21 aa (all unique) mutation sites were identified in the Hungarian L sequences from which $11 \mathrm{nt}$ mutation sites were detected in at least two strains. Six out of 11 common nt sites appeared in the L segment of strains SBV-Hun1, SBV-Hun3, SBV-Hun5, and SBV-Hun6 and five of those (nt583, nt768, nt1770, nt3315, and nt5813) were unique for all these strains also causing aa alteration at three sites (D195N, M590I, and R1938K). Additional two unique mutation sites within the ORF were present in SBV-Hun3, SBV-Hun5, and SBV-Hun6 (nt2871), and in SBV-Hun3 and SBV-Hun5 (nt5693), respectively; the latter caused aa change (K1898R) as well. Synonymous $n t$ changes ( $3 / 11$ of the shared mutation sites) were found in the SBV-Hun4 and SBV-Hun7 sequences (nt1713, nt3564, and nt6750), and in nine other cattle and sheep reference sequences. The overall nt and aa identity values ranged between $99.6 \%-99.9 \%$ and $99.3 \%-99.9 \%$ among the $\mathrm{L}$ sequences of SBVs. Moreover, the L segment showed $92.9 \%-93.0 \%$ nt and $98.2 \%-98.4 \%$ aa identity with their closest relative, the Shamonda virus strain (accession number: HE795105).

The majority of nt substitutions seemed to have irregular distribution, but some mutation sites were common for various SBV sequences, originating sometimes from different geographical regions or hosts. For example, the cattle origin German strains BH132/14, BH119/14/1-2, and BH119/14/3-4 had 12 common mutation sites in the L segment, and also shared common substitutions in the M segment. These three German and some additional SBV strains, including the cattle origin strain, 200.2, from Lichtenstein, the cattle origin Swiss strains, 79.4, 91.1, 100.3, and 102.2, the sheep origin German strain, BH619/12, and the Hungarian strains, SBV-Hun4 and SBV-Hun7, also had common mutation sites in their $\mathrm{L}$ and $\mathrm{M}$ sequences. Common and unique mutation sites were observed in the SBV-Hun1, SBV-Hun3, SBV-Hun5, and SBV-Hun6 sequences at five (nt583, nt768, nt1770, nt3315, and nt5813), one (nt623) and one (nt276 in the N region and nt251 in the NSs region) sites in the L, M, and $\mathrm{S}$ sequences, respectively, and at some sites, the same mutations were found in the sequence of the cattle origin Swiss strain 96.1 (at nt2190 and nt1569 in the L and $M$ segments, respectively). Some unique nt mutations of the SBV-Hun1, SBV-Hun3, SBV-Hun5, and SBV-Hun6 sequences caused aa changes at three (nt583 - aaD195N, nt1770 - aaM590I, and nt5813 - aaR1938K), one (nt623 - aaT208M), and one (nt251 - aaH84R in the NSs region of the S segment) sites in the deduced aa sequences. Multiple mutation sites were identified more often in the sheep origin $\mathrm{M}$ sequences, but the position and the number of these mutations were scattered. 
Phylogenetic analyses of the SBV genomic segments resulted in low resolution trees with low bootstrap values and the sequences clustered fairly close to each other, probably due to the irregular distribution of nt mutations. Despite that, some sequences, e.g., those of the Hungarian strains SBV-Hun1, SBV-Hun3, SBV-Hun5, and SBV-Hun6, grouped together on separated branches of the phylogenetic trees. The three other Hungarian SBV strains did not show a consistent grouping on the phylogenetic trees. These results are consistent with multiple introductions of SBV strains during 2014; however, no deeper insight was permitted to draw valid conclusions for an epidemiological evaluation, given the lack of transparency regarding animal movements that accompanies commercial sales and purchases. Although previous studies indicated that the mutations were independent of the host species [5], with the new genome sequence data reported in this study, the cattle and sheep origin $M$ sequences seem to occupy different positions in the $M$ tree suggesting that, as sequence information increases over time, new insight into host species evolution and adaptation mechanisms will be enabled (Figure 1).

\section{Discussion}

Over the past 5 years, an extended need for livestock production has been noticed in Hungary; with nearly $20 \%$ increase of the cattle (from 682,000 to $818,000)$ and a relatively constant number of sheep stocks (1.120-1.214 million animals) (www.ksh.hu). SBV infection rapidly spread throughout the European continent from 2011 onward [12] affecting the Hungarian cattle population as well.

To date, 11 full SBV genomic sequences were available in GenBank, originating from Germany, the Netherlands, and Belgium [3, 12, 27-29]. To investigate the variability of the SBV sequences, we characterized the coding sequence of seven SBV strains collected in Hungary, including the virus from the first local case detected in 2012 (SBV-Hun4), and six additional strains from samples collected in 2014 .

Previous studies described high mutation density, a mutation "hot spot," in an approximately 1,200 nt long fragment (nt 1,394-2,562) within the M segment $[5,27]$. The product of the NSs gene and M segment polyprotein gene may have a role in the immune evasion of the virus that could be the reason for the variability $[5,7,9,10,27]$. It was also suggested that the truncated product of the NSs gene, detected in congenital infections of lamb may be the result of the low selective pressure that might be another factor in the accumulation of nt mutations $[5,30]$. Furthermore, the adaptation to the mammalian and insect host may influence 

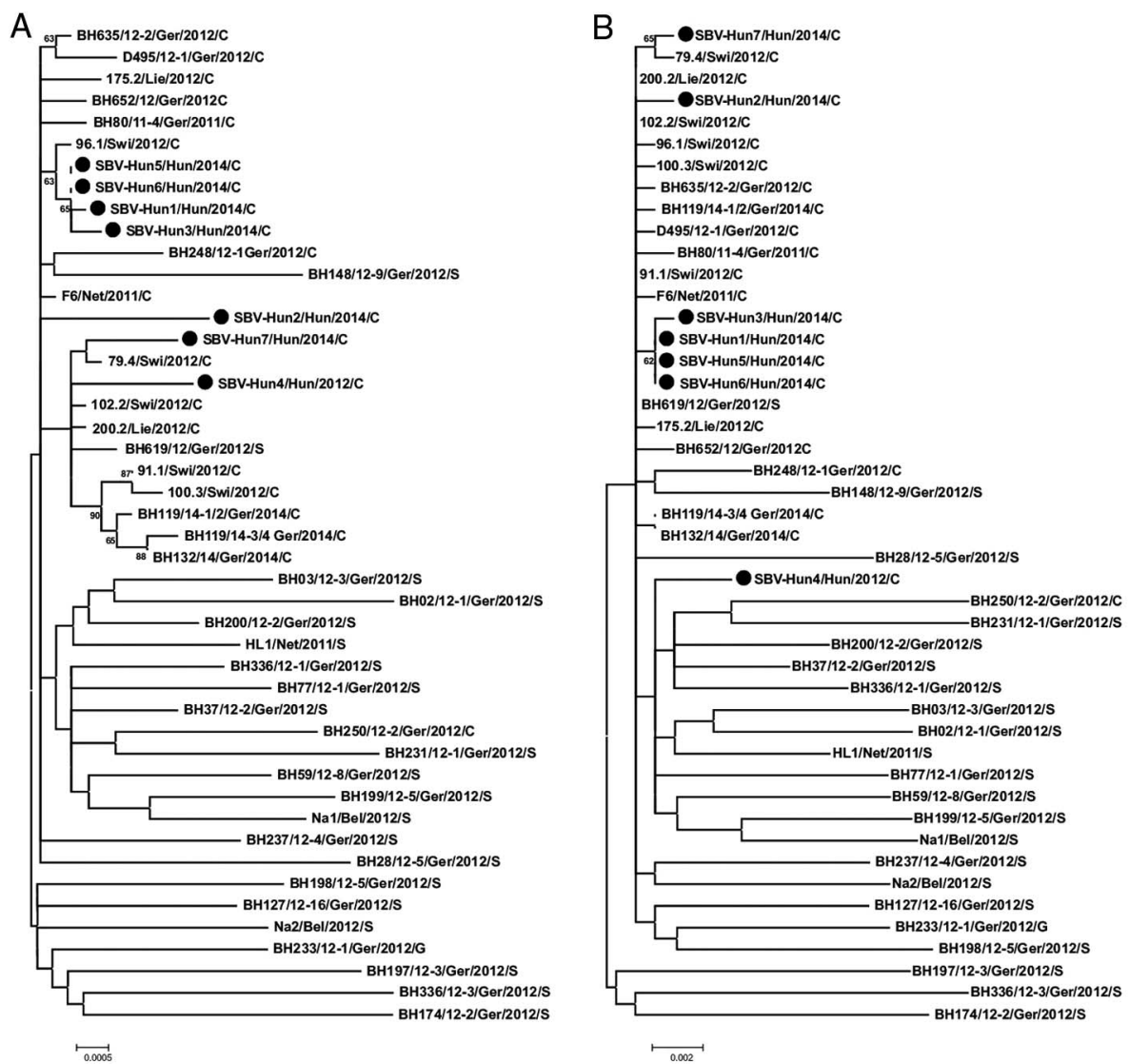

Figure 1. A. Nucleotide-based, unrooted maximum likelihood phylogenetic tree of the M segment polyprotein gene of SBVs. The phylogenetic tree was generated using the Tamura 3-parameter model and 500 bootstrap replicates. Bootstrap values $\geq 60$ are shown at the branch nodes. Hungarian SBV strains were labeled by black dots. Accession numbers of the SBV strains are listed in the "Materials and Methods" section and Table III. B. Amino acid-based, unrooted maximum likelihood phylogenetic tree of the polyprotein encoded by M segment. The phylogenetic tree was generated using the JTT model and 500 bootstrap replicates. Bootstrap values $\geq 60$ are shown at the branch nodes. Hungarian SBV isolates were labeled by black dots. Accession numbers of the SBV strains have been listed in the "Materials and Methods" section and Table III. C: cattle; S: sheep; G: goat

divergence within the viral genome [5]. The M segment of Hungarian SBV strains comprised 2-11 nt substitutions within the coding region, whereas only $1-5 \mathrm{nt}$ substitutions were described in the hypervariable region (nt 1,394-2,562) [27]. The bovine fetus origin Hungarian Ns, M sequence, and the M segment hypervariable (strain SBV-Hun4) region did not contain greater number of mutations than those of blood samples of acute cases from cattle. Thus, unlike those studies 
that reported accumulation of mutations in lamb fetus origin SBVs, we found no evidence that mutations would accumulate in cattle fetus origin SBVs [5, 30]. In contrast, differences were revealed when the $\mathrm{M}$ sequences were compared by host origin. In general, more nt mutations were detected for the sheep origin $\mathrm{M}$ polyprotein sequences (typically, 10-24 mutation sites and some indel mutations) compared with the Hungarian (2-11 nt mutation sites) and other cattle origin (typically, 1-9 nt mutation sites) SBV sequences. When comparing the hypervariable region of the $M$ sequences [27], the cattle origin sequences had typically 1-5 nt mutation sites, whereas the sheep origin sequences had typically 5-17 substitutions.

In summary, in this study, we characterized the coding sequence of seven Hungarian SBV strains and compared those with reference SBV sequences. The increasing number of whole genome sequences and the in vitro reverse genetic systems may facilitate the interpretation of genomic diversity and the role of the substitutions in the SBV genomes [7-10]. Even though high seroprevalence was detected in livestock [31], an accidental infection may compromise unprotected animals and help open the way to new epizootics to emerge.

\section{Acknowledgements}

The authors would like to acknowledge the excellent technical support provided by Ágnes Juhász and Ernőné Ottinger. KB was supported by the Momentum program, EF and SM were the recipients of the Bolyai Scholarship Program.

\section{Conflict of Interest}

The authors declare no conflict of interest.

\section{References}

1. Elliott, R. M.: Orthobunyaviruses: Recent genetic and structural insights. Nat Rev Microbiol 12, 673-685 (2014).

2. Goller, K. V., Höper, D., Schirrmeier, H., Mettenleiter, T. C., Beer, M.: Schmallenberg virus as possible ancestor of Shamonda virus. Emerg Infect Dis 18, 1644-1646 (2012).

3. Hoffmann, B., Scheuch, M., Höper, D., Jungblut, R., Holsteg, M., Schirrmeier, H., Eschbaumer, M., Goller, K. V., Wernike, K., Fischer, M., Breithaupt, A., Mettenleiter, T. C., Beer, M.: Novel orthobunyavirus in Cattle, Europe, 2011. Emerg Infect Dis 18, 469472 (2012).

4. Walter, C. T., Barr, J. N.: Recent advances in the molecular and cellular biology of bunyaviruses. J Gen Virol 92, 2467-2484 (2011). 
5. Fischer, M., Hoffmann, B., Goller, K. V., Hoper, D., Wernike, K., Beer, M.: A mutation 'hot spot' in the Schmallenberg virus M segment. J Gen Virol 94, 1161-1167 (2013).

6. Roman-Sosa, G., Brocchi, E., Schirrmeier, H., Wernike, K., Schelp, C., Beer, M.: Analysis of the humoral immune response against the envelope glycoprotein Gc of Schmallenberg virus reveals a domain located at the amino terminus targeted by mAbs with neutralizing activity. J Gen Virol 97, 571-580 (2016).

7. Varela, M., Pinto, R. M., Caporale, M., Piras, I. M., Taggart, A., Seehusen, F., Hahn, K., Janowicz, A., de Souza, W. M., Baumgärtner, W., Shi, X., Palmarini, M.: Mutations in the Schmallenberg virus Gc glycoprotein facilitate cellular protein synthesis shutoff and restore pathogenicity of NSs deletion mutants in mice. J Virol 90, 5440-5450 (2016).

8. Elliott, R. M., Blakqori, G., van Knippenberg, I. C., Koudriakova, E., Li, P., McLees, A., Shi, X., Szemiel, A. M.: Establishment of a reverse genetics system for Schmallenberg virus, a newly emerged orthobunyavirus in Europe. J Gen Virol 94, 851-859 (2013).

9. Kraatz, F., Wernike, K., Hechinger, S., König, P., Granzow, H., Reimann, I., Beer, M.: Deletion mutants of Schmallenberg virus are avirulent and protect from virus challenge. J Virol 89, 1825-1837 (2015).

10. Varela, M., Schnettler, E., Caporale, M., Murgia, C., Barry, G., McFarlane, M., McGregor, E., Piras, I. M., Shaw, A., Lamm, C., Janowicz, A., Beer, M., Glass, M., Herder, V., Hahn, K., Baumgärtner, W., Kohl, A., Palmarini, M.: Schmallenberg virus pathogenesis, tropism and interaction with the innate immune system of the host. PLoS Pathog 9, e1003133 (2013).

11. Wernike, K., Elbers, A., Beer, M.: Schmallenberg virus infection. Rev Sci Tech 34, 363-373 (2015a).

12. Wernike, K., Hoffmann, B., Conraths, F. J., Beer, M.: Schmallenberg virus recurrence, Germany, 2014. Emerg Infect Dis 21, 1202-1204 (2015b).

13. Chiari, M., Sozzi, E., Zanoni, M., Alborali, L. G., Lavazza, A., Cordioli, P.: Serosurvey for Schmallenberg virus in alpine wild ungulates. Transbound Emerg Dis 61, 1-3 (2013).

14. Larska, M., Krzysiak, M., Smreczak, M., Polak, M.P., Zmudziński, J. F.: First detection of Schmallenberg virus in elk (Alces alces) indicating infection of wildlife in Białowieża National Park in Poland. Vet J 198, 279-281 (2013).

15. Larska, M., Krzysiak, M. K., K sik-Maliszewska, J., Rola, J.: Cross-sectional study of Schmallenberg virus seroprevalence in wild ruminants in Poland at the end of the vector season of 2013. BMC Vet Res 10, 967 (2014).

16. Linden, A., Desmecht, D., Volpe, R., Wirtgen, M., Gregoire, F., Pirson, J., Paternostre, J., Kleijnen, D., Schirrmeier, H., Beer, M., Garigliany, M. M.: Epizootic spread of Schmallenberg virus among wild cervids, Belgium, fall 2011. Emerg Infect Dis 18, 2006-2008 (2012).

17. Mouchantat, S., Wernike, K., Lutz, W., Hoffmann, B., Ulrich, R. G., Börner, K., Wittstatt, U., Beer, M.: A broad spectrum screening of Schmallenberg virus antibodies in wildlife animals in Germany. Vet Res 46, 99 (2015).

18. van den Brom, R., Luttikholt, S. J., Lievaart-Peterson, K., Peperkamp, N. H., Mars, M. H., van der Poel, W. H., Vellema, P.: Epizootic of ovine congenital malformations associated with Schmallenberg virus infection. Tijdschr Diergeneeskd 137, 106-111 (2012).

19. Garigliany, M. M., Hoffmann, B., Dive, M., Sartelet, A., Bayrou, C., Cassart, D., Beer, M., Desmecht, D.: Schmallenberg virus in calf born at term with porencephaly, Belgium. Emerg Infect Dis 18, 1005-1006 (2012). 
20. Wernike, K., Conraths, F., Zanella, G., Granzow, H., Gache, K., Schirrmeier, H., Valas, S., Staubach, C., Marianneau, P., Kraatz, F., Höreth-Böntgen, D., Reimann, I., Zientara, S., Beer, M.: Schmallenberg virus - Two years of experiences. Prev Vet Med 116, 423-434 (2014).

21. De Regge, N., Deblauwe, I., De Deken, R., Vantieghem, P., Madder, M., Geysen, D., Smeets, F., Losson, B., van den Berg, T., Cay, A. B.: Detection of Schmallenberg virus in different Culicoides spp. by real-time RT-PCR. Transbound Emerg Dis 59, 471-475 (2012).

22. Elbers, A. R., Meiswinkel, R., van Weezep, E., Sloet van Oldruitenborgh-Oosterbaan, M. M., Kooi, E. A.: Schmallenberg virus in Culicoides spp. biting midges, the Netherlands, 2011. Emerg Infect Dis 19, 106-109 (2013).

23. Rasmussen, L. D., Kristensen, B., Kirkeby, C., Rasmussen, T. B., Belsham, G. J., Bodker, R., Botner, A.: Culicoids as vectors of Schmallenberg virus. Emerg Infect Dis 18, 1204-1206 (2012).

24. Bilk, S., Schulze, C., Fischer, M., Beer, M., Hlinak, A., Hoffmann, B.: Organ distribution of Schmallenberg virus RNA in malformed newborns. Vet Microbiol 159, 236-238 (2012).

25. Larsson, A.: AliView: A fast and lightweight alignment viewer and editor for large data sets. Bioinformatics 30, 3276-3278 (2014).

26. Tamura, K., Stecher, G., Peterson, D., Filipski, A., Kumar, S.: MEGA6: Molecular evolutionary genetics analysis version 6.0. Mol Biol Evol 30, 2725-2729 (2013).

27. Coupeau, D., Claine, F., Wiggers, L., Kirschvink, N., Muylkens, B.: In vivo and in vitro identification of a hypervariable region in Schmallenberg virus. J Gen Virol 94, 1168-1174 (2013).

28. Hulst, M., Kortekaas, J., Hakze-van der Honing, R., Vastenhouw, S., Cornellissen, J., van Maanen, K., Bossers, A., Harders, F., Stockhofe, N., van der Poel, W.: Genetic characterization of an atypical Schmallenberg virus isolated from the brain of a malformed lamb. Virus Genes 47, 505-514 (2013).

29. Hofmann, M. A., Mader, M., Flückiger, F., Renzullo, S.: Genetic stability of Schmallenberg virus in vivo during an epidemic, and in vitro, when passaged in the highly susceptible porcine SK-6 cell line. Vet Microbiol 176, 97-108 (2015).

30. Coupeau, D., Claine, F., Wiggers, L., Kirschvink, N., Muylkens, B.: S segment variability during the two first years of the spread of Schmallenberg virus. Arch Virol 161, 1353-1358 (2016).

31. Elbers, A. R., Loeffen, W. L., Quak, S., de Boer-Luijtze, E., van der Spek, A. N., Bouwstra, R., Maas, R., Spierenburg, M. A., de Kluijver, E. P., van Schaik, G., van der Poel, W. H.: Seroprevalence of Schmallenberg virus antibodies among dairy cattle, the Netherlands, winter 2011-2012. Emerg Infect Dis 18, 1065-1071 (2012). 